\title{
РЕКУРРЕНТНОЕ МОДЕЛИРОВАНИЕ АВТОРЕГРЕССИИ МНОГОМЕРНЫХ ПО ВХОДУ И ВЫХОДУ РАЗНОГО ПОРЯДКА ЛИНЕЙНЫХ ДИСКРЕТНЫХ ДИНАМИЧЕСКИХ СИСТЕМ ПРИ НАЛИЧИИ АВТОКОРРЕЛИРОВАННЫХ ПОМЕХ В ВЫХОДНЫХ СИГНАЛАХ
}

\author{
И. Л. Сандлер \\ Самарский государственньй университет путей сообщения
}

Поступила в редакцию 24.10.2017 г.

\begin{abstract}
Аннотация. Рассматривается методика параметрической идентификации линейной дискретной динамической системы многомерной по входу и выходу, имеющая разный порядок входных и выходных сигналах с автокоррелированными помехами в выходных сигналах, описываемой авторегрессинной моделью. Доказывается сильная состоятельность оценок неизвестных параметров при помощи стохастического градиентного алгоритма, практические результаты доказывают эффективность сходимости предложенного алгоритма.

Ключевые слова: авторегрессионная модель ЛДС, рекуррентный алгоритм моделирования, параметрическая идентификация, модель линейной динамической системы, автокоррелированные помехи, нелинейный метод наименьших квадратов.

Annotation. The technique of parametrical identification of linear discrete dynamic system multidimensional input and output with a different order of the input and output signals with autocorrelated interference in the output signals described autoregressive model. We prove strong consistency of estimates of the unknown parameters using the stochastic gradient algorithm, the practical results prove the efficiency of convergence of the proposed algorithm.

Keywords: autoregressive model of LDS, recurrent simulation algorithm, parametric identification, linear dynamic model of the system, autocorrelated noise, the nonlinear least squares method.
\end{abstract}

\section{ВВЕДЕНИЕ}

Применение методов позволяющие идентифицировать сложные объекты управления в наше время имеют довольно большую разновидность, однако каждый рассматриваемый алгоритм идентификации для конкретной задачи может иметь свои недостатки: низкую точность оценивания, отсутствием информации о внешней среде и связях между параметрами, высокую вычислительную сложность, недостаточную скорость сходимости, отсутствие учета помех в наблюдаемых сигналах, и как следствие повышается несостоятельность. Поэтому, разработка новых методов идентификации, совмещающие

() Сандлер И. Л., 2018 условия состоятельности, скорости сходимости, малой априорной информации, учета сложных помех, является актуальной задачей. В работах [1-6] рассматривается посторенние рекуррентного алгоритма оценивания параметров многомерной (многосвязной) дискретной ЛДС разного порядка при наличии белошумных помех наблюдений.

В работе рассматривается рекуррентный алгоритм, который позволяет получать сильно состоятельные оценки неизвестных параметров многомерной по входу и выходу дискретной разного порядка ЛДС описываемая авторегрессинной моделью при наличии автокоррелированных помех в выходных сигналах ЛДС, на основе стохастического градиентного алгоритма. 
Рекуррентное моделирование авторегрессии многомерных по входу и выходу ...

\section{ПОСТАНОВКА ЗАДАЧИ}

Дискретная линейная динамическая система, описывается следующим уравнением:

$$
\begin{gathered}
z_{i}^{(n)}-\sum_{m=1}^{\bar{r}_{m n}} b_{0}^{(m n)}(n) z_{i-m}^{(n)}= \\
=\sum_{\substack{l=1 \\
k \neq n}}^{k} \sum_{m=1}^{\bar{r}_{n l}} b_{0}^{(m l)}(n) z_{i-m}^{(l)}+\xi_{2}^{(n)}(i) \\
y_{i}^{(l)}=z_{i}^{(l)}+\xi_{1}^{(l)}(i),
\end{gathered}
$$

где $n=\overline{1, k}, y_{i}^{(l)}$ - наблюдаемые выходные сигналы динамической системы; $z_{i}^{(l)}-$ ненаблюдаемые выходные сигналы динамической системы, $l=\overline{1, k} ; k$ - число выходных переменных; $b_{0}^{(m l)}(n)$ - параметры; $\xi_{1}^{(l)}(i)$ - помеха наблюдений в l-м выходном сигнале динамической системы;

Пусть матрица $\Xi_{\bar{r}_{n k}}$ имеет вид

$$
\begin{aligned}
\Xi_{\bar{r}_{n k}}= & \left(\xi_{1}^{(n)}(i) \vdots \Xi_{\bar{r}_{n n}}^{T}(i-1) \vdots \Xi_{\bar{r}_{n 1}}^{T}(i-1) \vdots \ldots\right. \\
& \left.\vdots \Xi_{\bar{r}_{n k}}^{T}(i-1)\right)^{T} \in R_{\sum_{i=1} \bar{r}_{\bar{r}_{l}}+1} ;
\end{aligned}
$$

Тогда

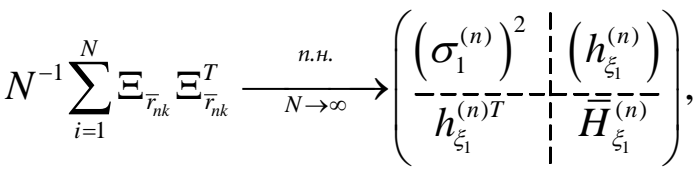

$$
\begin{aligned}
& h_{\xi_{1}}^{(n)}: 1 \times \sum_{l=1}^{k} \bar{r}_{n l},
\end{aligned}
$$$$
\bar{H}_{\xi_{1}}^{(n)}=\left(\begin{array}{c:c:c:c}
H_{\xi_{1}}^{(n)} & 0 & \cdots & 0 \\
\hdashline 0 & H_{\xi_{1}}^{(1)} & \cdots & \vdots \\
\hdashline \vdots & \cdots & \ddots & \vdots \\
\hdashline 0 & \cdots & \cdots & H_{\xi_{1}}^{(k)}
\end{array}\right),
$$$$
H_{\xi_{1}}^{(l)}=\left(\begin{array}{ccc}
h_{\xi_{1}}^{(l)}(0) & \cdots & h_{\xi_{1}}^{(l)}\left(r_{l}-1\right) \\
\vdots & & \vdots \\
h_{\xi_{1}}^{(l)}\left(r_{l}-1\right) & \cdots & h_{\xi_{1}}^{(l)}(0)
\end{array}\right) \text {, }
$$

$H_{\xi_{1}}^{(n)}: \sum_{l=1}^{k} \bar{r}_{n l} \times \sum_{l=1}^{k} \bar{r}_{n l}-$ множество вещественных матриц определенного порядка.

Для разработки методики параметрической идентификации допустим выполнение следующих условий:
$1^{0}$. Истинные значения параметров устойчивой линейной системы априорно принадлежащие множеству $\tilde{\mathrm{B}}$, являются компактом.

$2^{0}$. Последовательности помех наблюдений в 1-м выходном сигнале $\left\{\xi_{1}^{(l)}(i)\right\}$, и входной белый шум $\left\{\xi_{2}^{(n)}(i)\right\}$ статистически независимые последовательности и стационарные в совокупности в узком смысле с

$$
\begin{gathered}
E\left(\xi_{1}^{(l)}(i+1) / F_{1}^{(l)}\right)=0, E\left(\xi_{2}^{(n)}(i+1) / F_{2}^{(n)}\right)=0, \\
E\left[\left\{\xi_{1}^{(l)}(i+1)\right\}^{2} / F_{1}^{(l)}\right] \leq W_{1}^{(l)}(i),
\end{gathered}
$$

$E\left[\left\{\xi_{2}^{(n)}(i+1)\right\}^{2} / F_{2}^{(n)}\right] \leq W_{2}^{(n)}(i)$, где $F_{1}^{(l)}, F_{2}^{(n)}-$ $\sigma$-алгебры индуцированные семействами случайных величин $\left\{\xi_{1}^{(l)}\left(i_{0}\right), \ldots, \xi_{1}^{(l)}(i)\right\}$, $\left\{\xi_{2}^{(n)}\left(i_{0}\right), \ldots, \xi_{2}^{(n)}(i)\right\} ; W_{1}^{(l)}(i), W_{2}^{(n)}(i)-$ случайные величины - $E\left(W_{1}^{(l)}(i)\right) \leq \pi_{\xi_{1}^{(l)}}$, $E\left(W_{2}^{(n)}(i)\right) \leq \pi_{\xi_{2}^{(n)}}$ с $l=\overline{1, k}$, где $E-$ оператор МО. Причем входной белый шум $\left\{\xi_{2}^{(n)}(i)\right\}$ имеет дробно-рациональную плотность.

$3^{0}$. Пусть

$N^{-1} \sum_{i=1}^{N} \xi_{1}^{(l)}(i) \xi_{1}^{(l)}(i+m) \underset{N \rightarrow \infty}{\longrightarrow} h_{\xi_{1}}^{(l)}(m)<\infty$ П.Н.,

$4^{0}$. Выполняются условие несократимости полиномов

$$
B^{(n)}\left(q^{-1}\right)=1-\sum_{m=1}^{\bar{r}_{m n}} b_{0}^{(m n)} \cdot q^{-m},
$$

$\hat{A}^{(l)}\left(q^{-1}\right)=\sum_{m=1}^{\bar{r}_{n l}} b_{0}^{(m l)} \cdot q^{-m}$, где, $q^{-1}$ - оператор сдвига назад, $q^{-1} y_{i}=y_{i-1}$

$$
5^{0} \text {. Пусть } \gamma^{(n)}=h_{\xi_{2}}^{(n)}(0) / h_{\xi_{1}}^{(n)}(0) .
$$

Задачу параметрической идентификации можно сформулировать следующим образом: требуется рекуррентно определить оценки неизвестных параметров дискретной линейной динамической системы, описываемая уравнением (1) по наблюдаемым последовательностям $\left\{y_{i}^{(l)}\right\}$. 


\section{И. Л. Сандлер}

МЕТОДИКА АЛГОРИТМА

ПАРАМЕТРИЧЕСКОЙ

ИДЕНТИФИКАЦИИ ПАРАМЕТРОВ АВ-

ТОРЕГРЕССИОННОЙ МОДЕЛИ

МНОГОМЕРНОЙ ДИСКРЕТНОЙ

РАЗНОГО ПОРЯДКА ЛДС

С АВТОКОРРЕЛИРОВАННЫМИ

ПОМЕХАМИ

Используя критерий, при котором оценки будут сильно состоятельные, показанный в работе [7]:

$$
\min _{\left(\frac{b(n)}{a(n)}\right) \in \tilde{B}} \frac{\lim _{i \rightarrow \infty} E\left(y_{i}^{(n)}-(b(n))^{T}\left(Y_{\bar{F}_{n k}}(i-1)\right)\right)^{2}}{\bar{H}_{\xi_{1}}^{(n)}+b(n) \frac{\bar{\xi}_{\xi_{1}}}{\left(\sigma_{1}^{(n)}\right)^{2}}(n) b(n)^{T}-2 \frac{h_{\xi_{1}}}{\left(\sigma_{1}^{(n)}\right)^{2}} b(n)},
$$

где $b(n)=\left(\left(b^{(n)}(n)\right)^{T} \vdots \ldots:\left(b^{(k)}(n)\right)^{T}\right)^{T}$,

$$
\begin{aligned}
& b^{(l)}(n)=\left(b^{(1 l)}(n), \ldots, b^{\left(\bar{r}_{l l} l\right)}(n)\right)^{T}, \\
& Y_{\bar{r}_{n k}}(i-1)=\left(y_{\bar{r}_{n n}}^{(n) T} \vdots \ldots . \vdots y_{\bar{r}_{n k}}^{(k) T}\right)^{T}, \\
& y_{\bar{r}_{n l}}^{(l)}(i)=\left(y_{i-1}^{(l)}, \ldots y_{i-\bar{r}_{n l}}^{(l)}\right)^{T},
\end{aligned}
$$

Можно показать, что оценки неизвестного вектора параметров $\left(\frac{\hat{b}_{i}(n)}{\hat{a}_{i}-(n)}\right)$ можно получить с помощью стохастического градиентного алгоритма:

$$
\begin{gathered}
\left|\hat{b}_{i+1}(n)\right|= \\
=\left|\hat{b}_{i}(n)\right|-\alpha_{i} \nabla_{|b(n)|}\left[\frac{\left(y_{i+1}^{(n)}-\left|\hat{b}_{i}(n)\right|^{T}\left|Y_{\bar{r}_{k k}}(i+1)\right|\right)^{2}}{\omega\left(\hat{b}_{i}(n)\right)}\right],
\end{gathered}
$$

где $\omega\left(\hat{b}_{i}(n)\right)=1+\gamma^{(n)}+b(n) \frac{\bar{H}_{\xi_{1}}^{(n)}}{\left(\sigma_{1}^{(n)}\right)^{2}}(n) b(n)^{T}-$ $-2 \frac{h_{\xi_{1}}(n)}{\left(\sigma_{1}^{(n)}\right)^{2}} b(n)$

$\alpha_{i}$ - последовательность, для которой выполняется условия $\sum_{i=0}^{\infty} \alpha_{i}=\infty, \alpha_{i} \geq \alpha_{i+1}, \quad$ и $\sum_{i=0}^{\infty} \alpha_{i}^{t}<\infty$ при $t>1\left(6^{0}\right)$ и $\sum_{i=1}^{\infty} \alpha_{i} \xi_{1}^{(l)}(i)<\infty$, п.н. $\left(7^{0}\right)$

Теорема состоятельности алгоритма (3).

Пусть авторегрессионная модель многомерной дискретной разного порядка лдс с ав- токоррелированными помехами описываемая уравнением (1) и при выполнении условий $1^{0}-7^{0}$ оценки, определяемые рекуррентным алгоритмом (3) либо стремятся к истинным значениям $\left|\hat{b}_{i}(n)\right| \longrightarrow \underset{i \rightarrow \infty}{\longrightarrow}\left|b_{0}(n)\right|$ п.н, либо бесконечны $\left|\hat{b}_{i}(n)\right| \underset{i \rightarrow \infty}{\longrightarrow} \infty$.

Доказательство теоремы состоятельности получаемых с помощью алгоритма (3) оценок базируется на методе непрерывных моделей [8]. Построим асимптотическую непрерывную детерминируемую модель алгоритма (3). Функционал (2) представим в виде:

$$
\begin{gathered}
J(b(n))=\left(\sigma_{1}^{(n)}\right)^{2}+ \\
+\frac{\left(|b(n)|-\left|b_{0}(n)\right|\right)^{T} H^{*}\left(|b(n)|-\left|b_{0}(n)\right|\right)}{\omega\left(\hat{b}_{i}(n)\right)}
\end{gathered}
$$

при $|b(n)| \in R_{\bar{r}_{n n}+\bar{r}_{n 1}+\ldots+\bar{r}_{n k}}$ где,

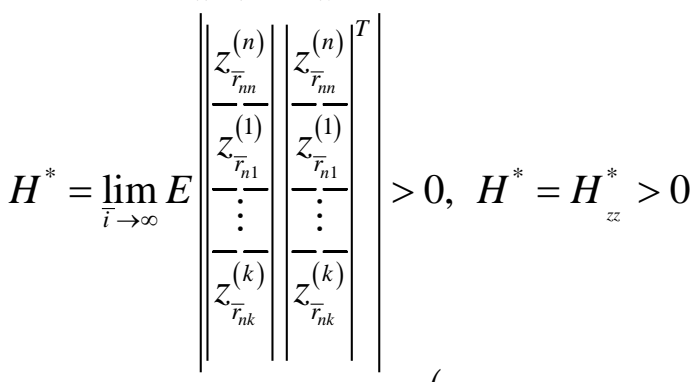

матрица порядка $\left(\bar{r}_{n n}+\bar{r}_{n 1}+\ldots+\bar{r}_{n k}\right) \times$ $\times\left(\bar{r}_{n n}+\bar{r}_{n 1}+\ldots \bar{r}_{n k}\right)$, что следует из $1^{0}, 4^{0}, 6^{0}$, где, $Z_{\bar{r}_{n l}}^{(l)}(i)=\left|z_{i-1}^{(l)}, \ldots, z_{i-\bar{r}_{n l}}^{(l)}\right|^{T}-$ вектор $\bar{r}_{n l} \times 1$.

Тогда асимптотическая непрерывная детерминированная модель:

$$
b(\dot{n})=-\nabla_{b(n)} J(b(n))
$$

и функция Ляпунова:

$$
V(b(n))=J(b(n)),
$$

дифференцируема и

$$
\begin{aligned}
& \dot{V}(b(n))=\nabla_{(b(n))}^{T} V(b(n)) \nabla_{(b(n))} J(b(n))= \\
&=-\left\|\nabla_{(b(n))} J(b(n))\right\|^{2}, \\
& \nabla_{(b(n))}^{T} V(b(n)) J(b(n))<0
\end{aligned}
$$

то $\tilde{B}=\left\{(b(n)) \in R_{\left(\bar{r}_{n n}+\bar{r}_{n 1}+\ldots+\bar{r}_{n k}\right)}: \dot{V}(b(n))=0\right\}$, coстоит из стационарных точек $J(b(n))$ [7]. При переходе от (3) к непрерывной модели необходимо показать, что для последователь- 
Рекуррентное моделирование авторегрессии многомерных по входу и выходу ...

ностей $\left\{\xi_{1}^{(l)}(i)\right\},\left\{\xi_{2}^{(n)}(i)\right\}$ и $\left\{\alpha_{i}\right\}$ выполняется равенство [8]:

$$
\begin{gathered}
\lim _{T \rightarrow 0} \limsup _{t \rightarrow \infty} \frac{1}{T} \| \sum_{i=t}^{m(t, T)} \alpha_{i} e_{i}\left((\hat{b}(i)),\left\{\xi_{1}^{(l)}(i)\right\},\right. \\
\left.\left\{\xi_{2}^{(n)}(i)\right\}\right) \|=0,
\end{gathered}
$$

где для $m(t, T)=\max \left\{m: \sum_{i=t}^{m} \alpha_{i} \leq T\right\}\|\cdot\|-$ евклидова норма. Для (6) необходимо ограниченность $\{(\hat{b}(i))\}$, что подразумевает ограниченность роста функции $\nabla_{(b(n))} J(b(n))$ при $\|b(n)\| \longrightarrow \infty$. Тогда $\lim _{\|b(n)\| \rightarrow \infty} \nabla_{(b(n))} J(b(n))=0$. Из ограниченности алгебраических сумм в условии $8^{0}$ и $\{(\hat{b}(i))\}$ следует ограниченность алгебраической

$\sum_{i=1}^{\infty} \alpha_{i} e_{i}\left((\hat{b}(i)),\left\{\xi_{1}^{(l)}(i)\right\},\left\{\xi_{2}^{(n)}(i)\right\}\right)<\infty$

суммы следует выполнение равенства (6).

Из теорем [8, с. 12, с. 292], следует, что при выполнении $1^{0}-7^{0}$ и (5) (6) $\{(\hat{b}(i))\}$ ограничена и при $i \rightarrow \infty,\{(\hat{b}(i))\}$ стремится к точкам множества $\tilde{B}$. Исследуя асимптотическую непрерывную детерминированную модель (4), можно показать, что $B_{*}=\left\{(b(n)) \in R_{\left(\bar{r}_{n n}+\bar{r}_{n 1}+\ldots+\bar{r}_{n k}\right)}:(b(n))=(b(n))\right\}$ состоит из одной единственной точки $\left(b_{0}(n)\right)$. Ход доказательства функции (7) теоремы, аналогичен доказательству приведенное в [6].

$$
\begin{aligned}
& J^{\prime}(u)=\frac{u^{T} H_{1}^{*} u}{u^{T} H_{\left(\bar{r}_{n n}+\bar{r}_{n 1}+\ldots+\bar{r}_{n k}+1\right)}^{\prime} u}, \\
& \text { где, } u=\left|u_{1}, \ldots, u_{\bar{r}_{n n}+\bar{r}_{n 1}+\ldots+\bar{r}_{n k}+1}\right|^{T} \in R_{\bar{r}_{n n}+\bar{r}_{11}+\ldots+\bar{r}_{n k}+1} \text {, } \\
& H_{1}^{*}=\lim _{i \rightarrow \infty} E\left[\left|\begin{array}{l}
-y_{i}^{(n)} \\
\left.\hdashline Y_{\bar{r}_{n k}}-\frac{1}{i}\right)
\end{array}\right|\left|-y_{i}^{(n)}: Y_{\bar{r}_{n k}}^{T}(i)\right|\right] \text {, } \\
& H_{\left(\bar{r}_{n n}+\bar{r}_{11}+\ldots+\bar{r}_{n k}+1\right)}^{\prime}=\left(\begin{array}{c:c} 
& \left(h_{\xi_{1}}^{(n)}\right)^{T} \\
1 & \left(\sigma_{1}^{(n)}\right)^{2} \\
\hdashline \frac{h_{\xi_{1}}^{(n)}}{\left(\sigma_{1}^{(n)}\right)^{2}} & \frac{\bar{H}_{\xi_{1}}^{(n)}}{\left(\sigma_{1}^{(n)}\right)^{2}}
\end{array}\right) .
\end{aligned}
$$

РЕЗУЛЬТАТЫ МОДЕЛИРОВАНИЯ АЛГОРИТМА ПАРАМЕТРИЧЕСКОЙ ИДЕНТИФИКАЦИИ ПАРАМЕТРОВ АВТОРЕГРЕССИОННОЙ МОДЕЛИ МНОГОМЕРНОЙ ДИСКРЕТНОЙ РАЗНОГО ПОРЯДКА ЛДС С АВТОКОРРЕЛИРОВАННЫМИ ПОМЕХАМИ

Разработанный стохастический градиентный алгоритм (3) программно реализован по средствам MatLab, для сравнительного анализа реализован рекуррентный метод инструментальных переменных и рекуррентный метод наименьших квадратов [13].

В примере многомерная разного порядка дискретная ЛДС описывалась следующими уравнениями:

$$
\begin{gathered}
z_{i}^{(1)}=1.2 z_{i-1}^{(1)}-0.7 z_{i-2}^{(1)}-0.3 z_{i-1}^{(2)}+1.2 z_{i-2}^{(2)}+\xi_{2}^{(1)}(i) \\
z_{i}^{(2)}=0.5 z_{i-1}^{(1)}-0.7 z_{i-2}^{(1)}-0.7 z_{i-1}^{(2)}+\xi_{2}^{(2)}(i) \\
y_{i}^{(l)}=z_{i}^{(l)}+\xi_{1}^{(l)}(i) .
\end{gathered}
$$

Число выходных переменных $k=2$, порядок выходных сигналов модели:

- по первому выходу $\bar{r}_{11}=2, \bar{r}_{12}=2$;

- по второму выходу $\bar{r}_{21}=2, \bar{r}_{22}=1$.

Входной сигнал в виде белого шума генерировался командой normrnd.

Помехи в выходных сигналах -

$$
\xi_{2}^{(k)}(i)=-0.5 \cdot \xi_{2}(i-1)-0.3 \cdot \xi_{2}(i-2)+e_{i},
$$

с дисперсией

$$
\sigma_{2}^{2}(i)=0.08+0.7 \cdot \sigma_{2}^{2}(i-1)+0.2 \cdot \xi_{2}^{2}(i-1) .
$$

Начальные значения параметров линейной динамической системы равны нулю. Отношение «сигнал-помеха» на выходе и выходе составляет 0.5. На рис. 1, 2 представлены графики погрешности оценок параметров для первого и второго выходов, определяемые как:

$$
=\sqrt{\left\|\left(\frac{\hat{b}_{i}(n)}{\hat{a}_{i}(n)}\right)-\left(\frac{b_{0}(n)}{a_{0}(n)}\right)\right\|^{2} / \|(n)=}
$$

Результаты относительных погрешностей оценок параметров многомерной разного порядка дискретной ЛДС, работы разработанного алгоритма (3) при различных 


\section{И. Л. Сандлер}

дисперсионных отношениях «помеха - сигнал» сведены в соответствующие диаграммы (рис. 3), наглядно поясняющие результаты.

На рис. 4 представлены графики относительных погрешностей моделирования ЛДС при различных отношениях «помеха - сигнал» для первого и второго выходов, определяемые как:

$$
\delta z_{N}(n)=\sqrt{\frac{\|\hat{z}(n)-z(n)\|^{2}}{\|z(n)\|^{2}}} \cdot 100 \%,
$$

где, $z(n)=\left(z_{1}(n), \ldots, z_{N}(n)\right)^{T}$ - вектор выходных ненаблюдаемых переменных; $\hat{z}(n)=\left(\hat{z}_{1}(n), \ldots, \hat{z}_{N}(n)\right)^{T}$ - оценка вектора выходных ненаблюдаемых переменных, полученная с помощью модели;

На рис. 5, 6 представлены входные сигналы, $z_{i}^{(1)}, z_{i}^{(2)}$ рассчитанные по истинным и оцененным параметрам при $i=10000$, $\sigma_{1}^{(n)} / \sigma_{z}^{(n)}=0,5$.

\section{ЗАКЛЮЧЕНИЕ}

Сравнение полученных оценок параметров дискретной динамической модели (1) с их истинными значениями при наличии ав- токоррелированных возмущений и [14-16], стохастический градиентный алгоритм (3) показывает высокую скорость сходимости, и точность оценивания в сравнении с классическими методами РМНК и РМИП. Графическое представление выходных сигналов $z_{i}^{(1)}$, $z_{i}^{(2)}$ динамической системы (рис. 5,6$)$ рассчитанные по истинным и идентифицированным параметрам при $i=10000, \sigma_{1}^{(n)} / \sigma_{z}^{(n)}=0,5$ показывает практически точное совпадение и соответствие выходных сигналов объекта модели, которые подтверждают эффективность работы алгоритма (3).

\section{СПИСОК ЛИТЕРАТУРЫ}

1. Кацюба О. А., Сандлер И. Л. Рекуррентное оценивание параметров многомерных по входу и выходу разного порядка линейных динамических систем с помехами во входных и выходных сигналах методом стохастической аппроксимации // Научно-технический журнал: «Системы управления и информационные технологии». - Москва-Воронеж, Научная книга, 2012. - Т. 47, № 1. - С. 31-34.

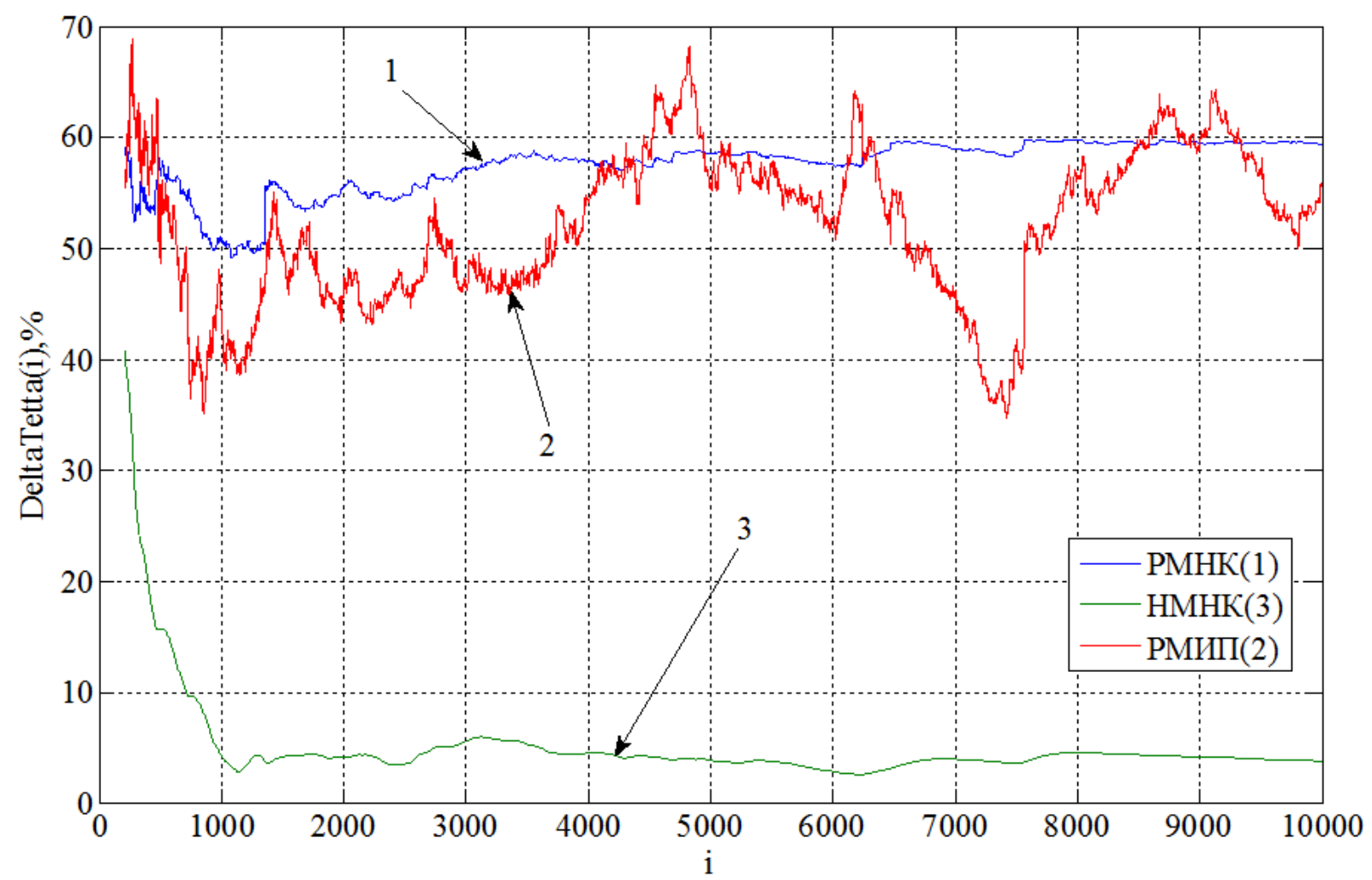

Рис. 1. Графики относительных погрешностей определения параметров для первого выхода 
Рекуррентное моделирование авторегрессии многомерных по входу и выходу ...

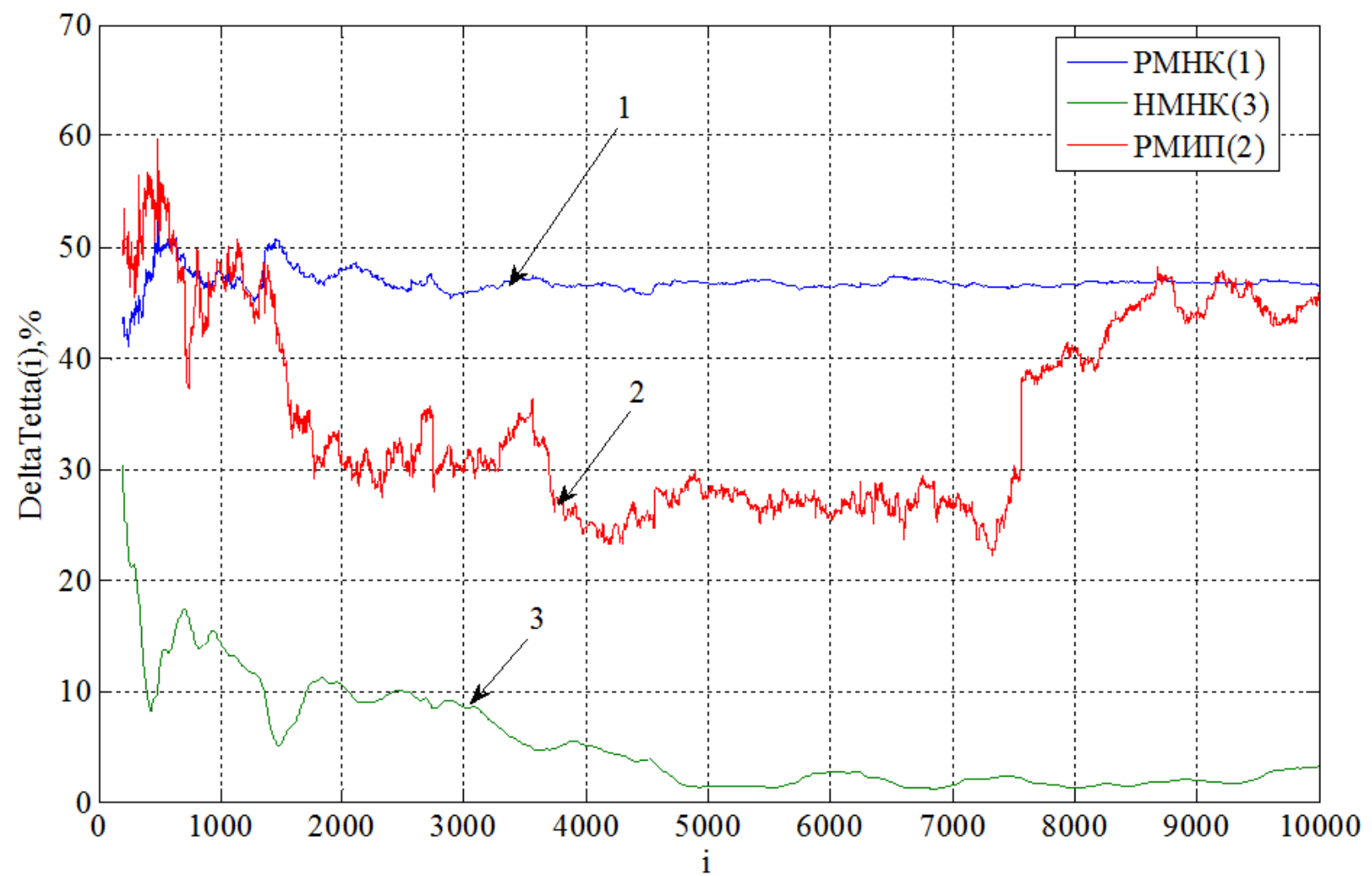

Рис. 2. Грабики относительных погрешностей определения параметров для второго выхода

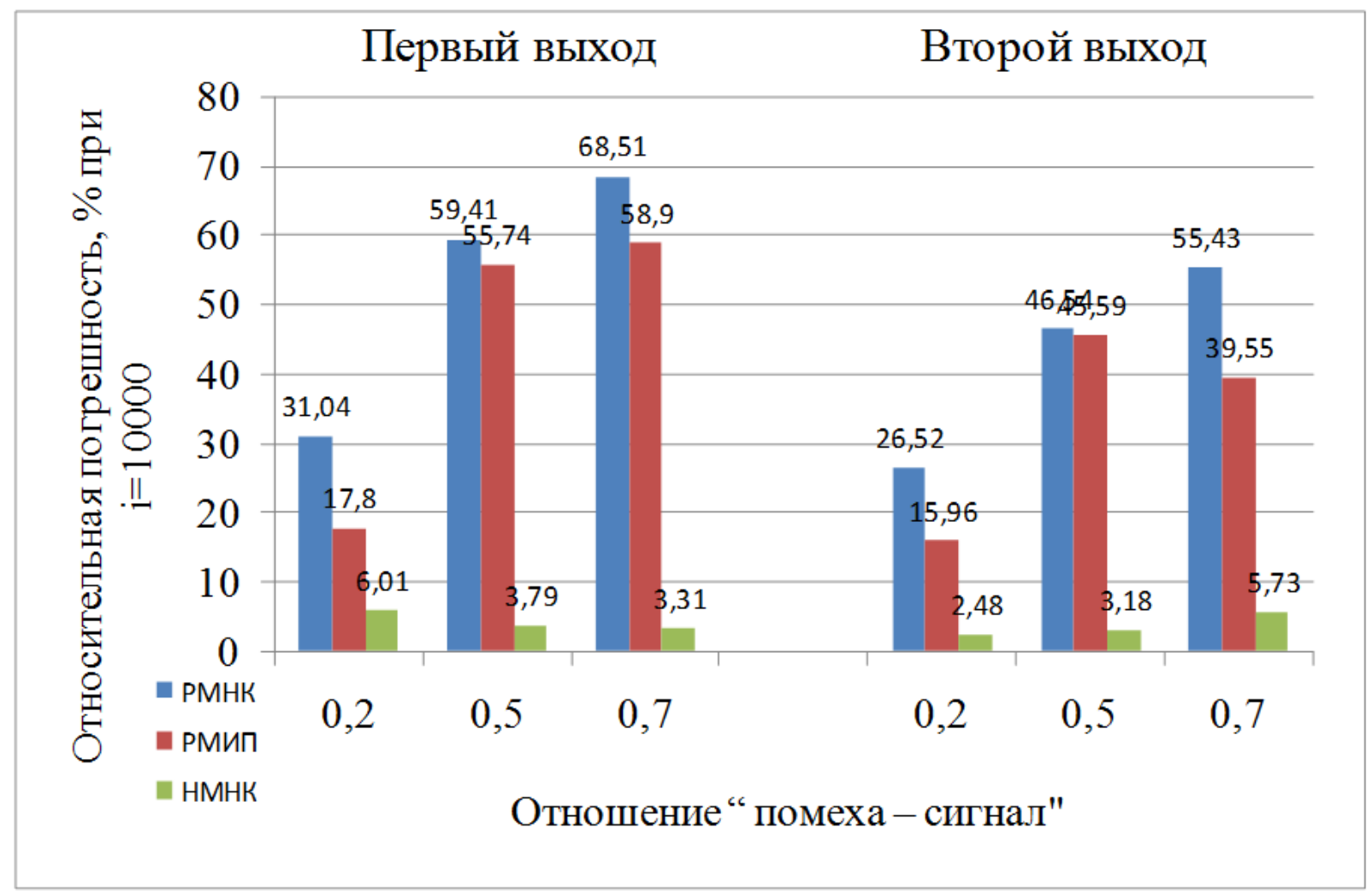

Рис. 3. Графики относительных погрешностей определения параметров ЛДС при различных отночениях «помеха - сигнал» 


\section{И. Л. Сандлер}

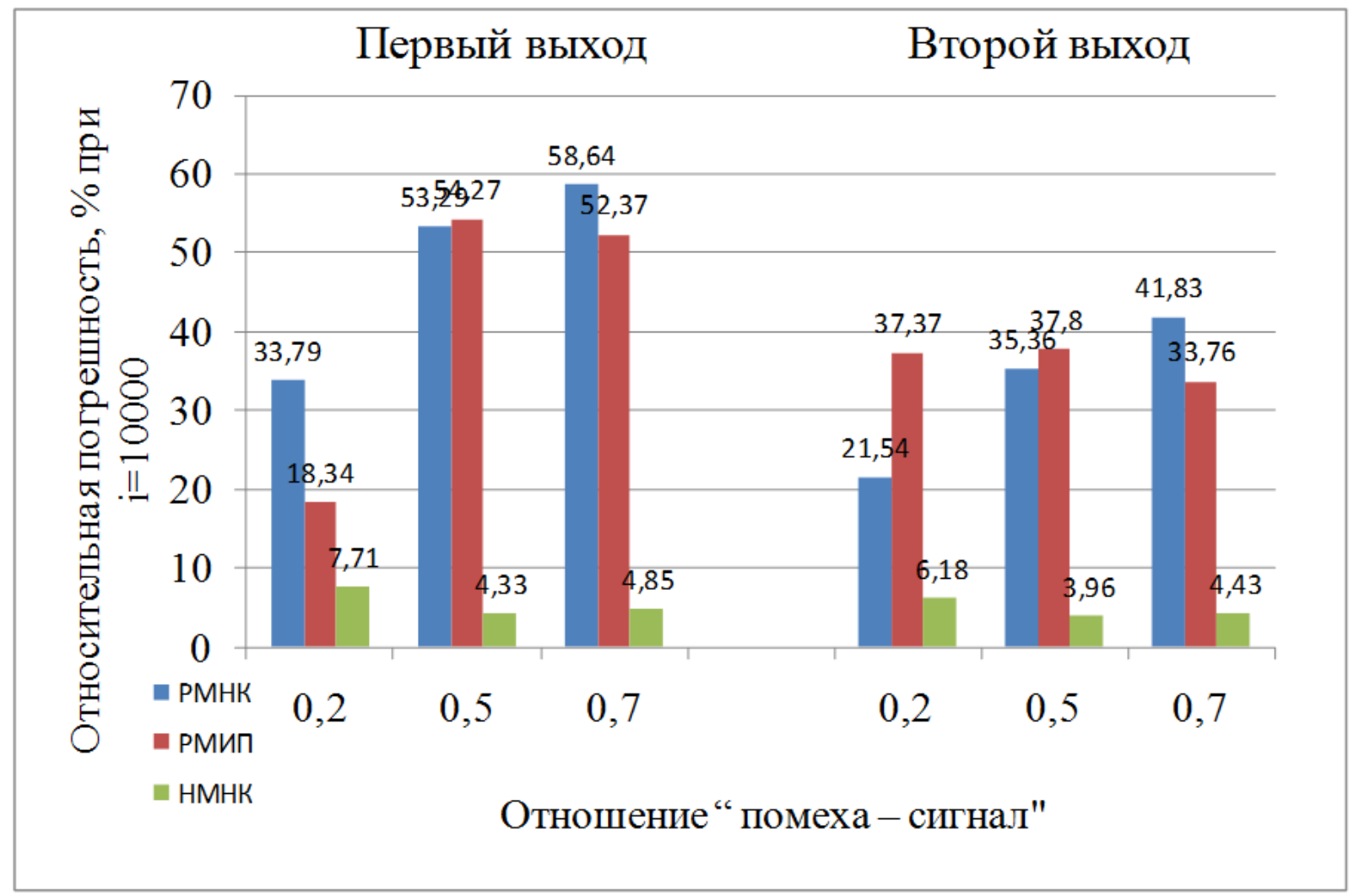

Рис. 4. Графики относительных погрешностей моделирования ЛДС при различных отношениях «помеха - сигнал»

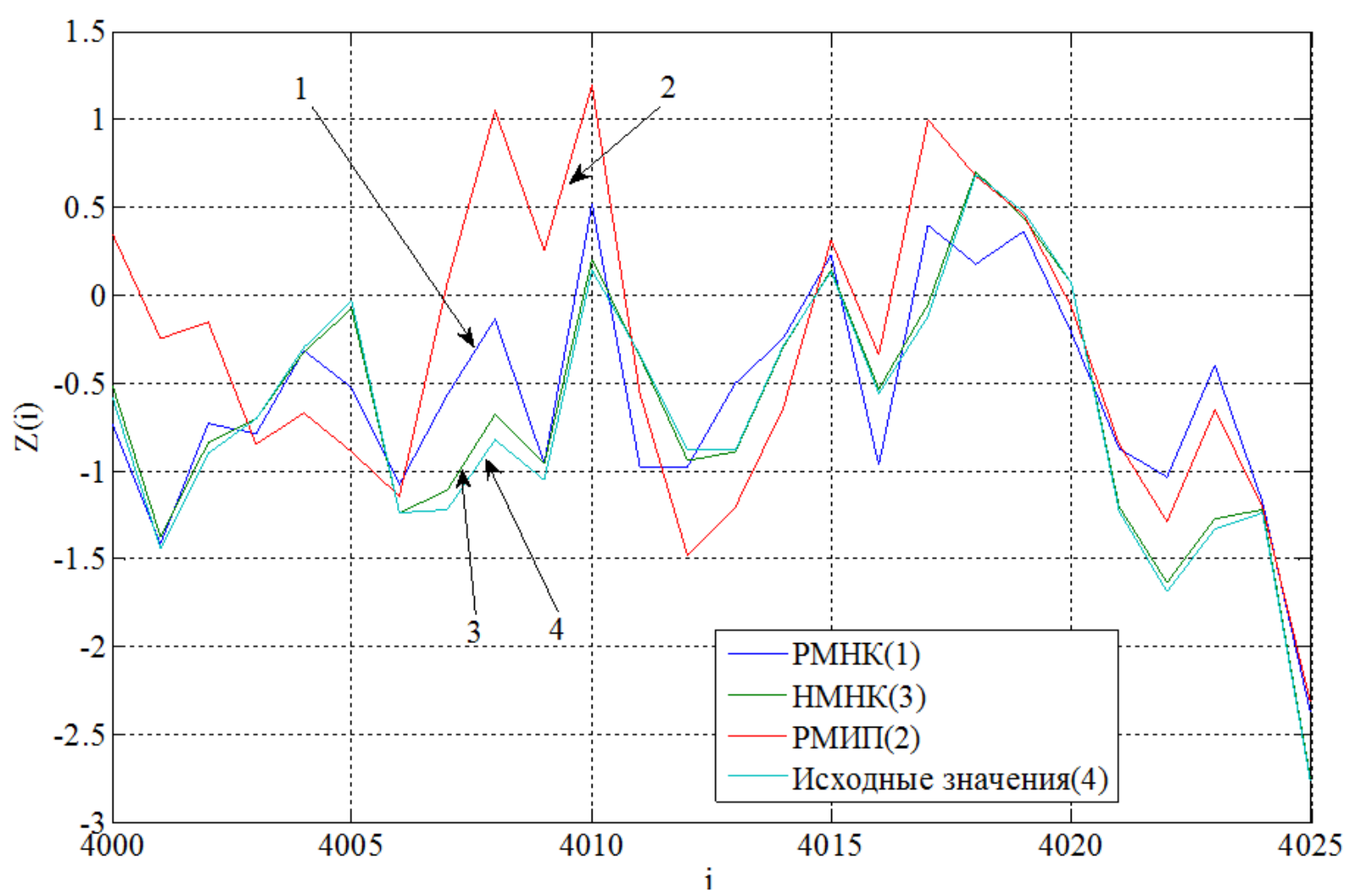

Рис. 5. $z_{i}^{(1)}$ рассчитанный по истинным и оцененным параметрам при $i=10000, \sigma_{1}^{(n)} / \sigma_{z}^{(n)}=0,5$ 
Рекуррентное моделирование авторегрессии многомерных по входу и выходу ...

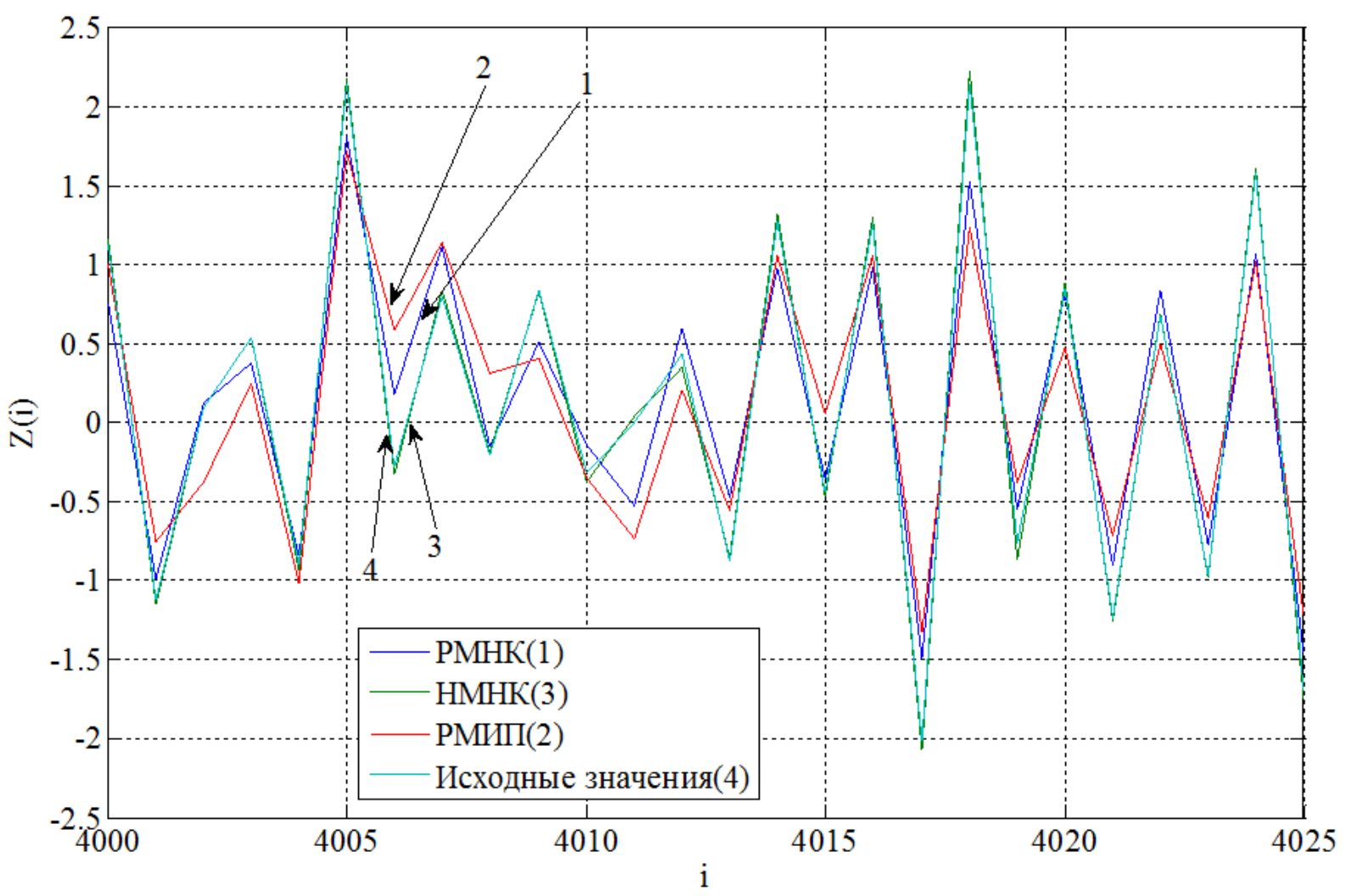

Рис. 6. $z_{i}^{(2)}$ рассчитанный по истинным и оцененным параметрам при $i=10000, \sigma_{1}^{(n)} / \sigma_{z}^{(n)}=0,5$

2. Сандлер И. Л. Рекуррентная идентификация параметров многомерных по входу и выходу разного порядка линейных динамических систем с помехами в выходных сигналах // Вестник транспорта Поволжья. 2012. - № 2(32). - С. 68-74.

3. Сандлер И. Л., Качюба О. А. Алгоритм рекуррентного оценивания параметров многомерных по входу и выходу разного порядка линейных динамических систем с ошибками в переменных // Перспективы развития информационных технологий. - 2011. - № 6. C. $49-52$.

4. Сандлер И. Л. Рекуррентное оценивание параметров многомерных линейных динамических систем с помехами наблюдений в выходных сигналах//Наука и образование транспорту. - 2014. - № 1. - С. 205-207.

5. Сандлер И. Л., Кацюба О. А. Рекуррентное оценивание параметров многомерных линейных динамических систем с ошибками по выходу // Математические методы в технике и технологиях - ММТT. - 2013. - № 2. C. 45-46.
6. Сандлер И. Л. Рекуррентное оценивание параметров авторегрессии многомерных по входу и выходу разного порядка линейных динамических систем с помехой в выходных сигналах методом стохастической аппроксимации// Системы управления и информационные технологии. - 2013. - Т. 53, № 3.1. C. $164-167$.

7. Кацюба О. А., Козлов Е. В. Оценивание параметров многосвязной линейной динамической системы разного порядка при наличии автокоррелированных помех во входных и выходных сигналах // Системы управления и информационные технологии. - Воронеж : ФГБОУ ВПО «Воронежский государственный технический университет», 2011. - № 3.1(45). C. 151-154.

8. Деревиикий Д. П., Фрадков А. Л. Прикладная теория дискретных адаптивных систем управления. - М. : Наука, 1991. - 215 с.

9. Chen H. F. Stochastic Approximation and Its Applications. Dordrecht: Kluwer, 2002. $357 \mathrm{p}$. 


\section{И. Л. Сандлер}

10. Гантмахер Ф. Р. Теория матриц. - М. : Наука, 1966. - 575 c.

11. Невельсон М. Б., Хасьминский Р. 3. Стохастическая аппроксимация и рекуррентное оценивание. - М. : Наука, 1972. - 304 с.

12. Chen H. F. A Unified Approach to Recursive System Identification/ In: Procc. $17^{\text {th }}$ IFAC Symposium on System Identification, Sait-Malo, France / World Congress, 17(Part 1), 2009. P. $420-425$.

13. Сандлер И. Л. Рекуррентное оценивание параметров авторегрессии многомерных по воду и выходу разного порядка линейных динамических систем с локально автокоррелированной помехой в выходном сигнале. Свидетельство о государственной регистрации программы для ЭВМ №2012660921. Дата регистрации 30.11.2012.

14. Сандлер И. Л. Тестирование рекуррентного алгоритма оценивания параметров многомерной линейной динамической системы с помехами // Актуальные направления науч-

Сандлер И. Л. - аспирант кафедры Мехатроника автоматизация и управление на транспорте, факультет Строительство железных дорог и информационные технологии, Самарский государственный университет путей сообщения.

E-mail: sandleri@bk.ru ных исследований XXI века: теория и практика, 2015. - Т. 3, № 5-2. - С. 117-121.

15. Сандлер И. Л. Тестирование рекуррентного алгоритма оценивания параметров многомерных по входу и выходу разного порядка линейных систем с автокоррелированными помехами во входных и выходных сигналах // Математическое и компьютерное моделирование естественнонаучных и социальных проблем IX Международная научно-техническая конференция молодых специалистов, аспирантов и студентов. Под редакцией И. В. Бойкова. - 2015. - С. 232-237.

16. Сандлер И. Л. Тестирование рекуррентного алгоритма оценивания параметров многомерных по входу и выходу разного порядка линейных систем с помехами // Identification systems. Theory and applications Proceedings of the International scientific and practical conference. Publishing House Science and Innovation Center, Ltd. - 2015. - C. 17-22.

Sandler I. L. - postgraduate student of the Department of mechatronics, automation and control in transport, faculty of Construction of Railways and information technology, Samara State Transport University.

E-mail: sandleri@bk.ru 